\title{
Psychologische Probleme bei den Transformationsprozessen in Ostdeutschland
}

\author{
Gisela Trommsdorff
}

In diesem Beitrag werden Schnittstellen zwischen sozio-politischem Wandel und psychologischen Prozessen herausgearbeitet. Zunächst wird erörtert, warum der Systemwechsel nach der Wende in Ostdeutschland und die darauf folgenden Transformationsprozesse mit psychologischen Problemen verbunden sind. Es wird gefragt, unter welchen Bedingungen der Systemumbruch für manche Menschen eher eine Belastung und für andere eher eine Chance bedeutet. Psychologische Theorieansätze werden hinsichtlich ihres Beitrages zur Analyse von individuellen Verarbeitungsformen der Transformationsprozesse erörtert. Individuelle Unterschiede in Belastungen hängen mit Sozialisationsbedingungen, Kohortenzugehörigkeit, Entwicklungsalter, gegenwärtigem sozialen Kontext, System- und Selbstbewertung zusammen. Transformationsbedingte Anpassungsprozesse werden im Hinblick auf Kontroll- und $\mathrm{Zu}-$ kunftsorientierung diskutiert. AbschlieBend werden methodische Probleme des Ost-West-Vergleichs behandelt.

\section{Systemwechsel und Transformation als psychologisches Problem}

\subsection{Soziologische und psychologische Aspekte der Transformation}

Mit dem Zusammenbruch der ehemaligen DDR und der Vereinigung beider deutscher Staaten fand in Ostdeutschland aus soziologischer Sicht ein vollständiger Systemwechsel statt; das politische und wirtschaftliche System und das Rechtswesen der alten Bundesrepublik wurden durch den Beitritt übernommen. Damit wurde abrupt ein Wechsel vom Sozialismus zur sozialen Marktwirtschaft und von totalitären zu demokratischen Verhältnissen vollzogen. Die damit entstandenen vielfältigen fundamentalen Umbrüche beschränken sich jedoch keineswegs auf den Austausch von Institutionen. Vielmehr vollziehen sich diese Umbrüche und ihre Nachwirkungen in einem grundlegenden Prozeß politischer, wirtschaftlicher, sozialer und kultureller Transformation, der wohl noch länger andauert. Inwieweit dieser Transformationsproze $\beta$ wiederum das inkorporierte System der alten Bundesrepublik verändert (soweit der Systemwechsel in den neuen Ländern Rückwirkungen auf die alten Länder hat), ob dies eine "nachholende", "komplementäre" oder "doppelte" Modernisierung bedeutet, und ob dies von Prozessen sozialen Wandels zu unterscheiden ist (vgl. Reißig, 1993; Zapf, 1993) - dies sind für die sozialwissenschaftliche Forschung und Theoriebildung wichtige Fragen, die im folgenden jedoch nicht im Vordergrund stehen sollen. Hier werden Transformationsprozesse als ein Teilbereich sozialen Wandels verstanden, die sich auf individuelles Handeln auswirken und durch psychologische Prozesse mit beeinflußt werden.

Der Verlauf der Transformation hängt u.a. von der Funktionsfähigkeit der ausgetauschten Institutionen und den damit verbundenen formalen Ordnungen und Organisationsstrukturen sowie auch deren Implementierung durch individuelles Verhalten ab. Daher ist zu fragen, ob der formale Systemwechsel mit den neuen Strukturen und Re- 
geln auch von den Menschen in den neuen Bundesländern akzeptiert wird, d.h. ob und inwieweit der Einzelne die entsprechenden, mit diesem System verbundenen Werte und Überzeugungen, Denk- und Verhaltensmuster zu übernehmen bereit ist. Damit geht es um die Menschen, die in diesen Institutionen handeln. Es sind Menschen, die diesen neuen Institutionen Legitimität zusprechen, die sie akzeptieren oder ablehnen können, und die die neuen Regeln durch entsprechende individuelle Deutungen, Entscheidungen und Kompetenzen mehr oder weniger angemessen umsetzen. Es sind Individuen, die in diesen Institutionen Aufgaben übernehmen und dazu bestimmte Fähigkeiten und Handlungsbereitschaften haben oder neu ausbilden. Daher beeinflußt individuelles Handeln direkt und indirekt die Funktionsweise und die Effizienz dieser formal inkorporierten aber subjektiv vielleicht als "oktroyiert" erlebten Institutionen. Dies wird z.B. sichtbar bei der Einrichtung und Leitung neuer Organisationen in Verwaltung und Wirtschaft, oder wenn es um die Auswahl und Schulung von Mitarbeitern und um die Zusammenarbeit mit ihnen geht (vgl. Reichardt \& Schröter, 1993; Wottawa, in diesem Band).

Darüber hinaus werden durch den Transformationsprozeß vielfaltige weitere individuelle Bewältigungsprozesse notwendig. Die Menschen der ehemaligen DDR sind in einem anderen System aufgewachsen und sozialisiert worden und haben Ziele, Motive, Wertschätzungen, Handlungserwartungen und -präferenzen sowie Gewohnheiten und Fähigkeiten entwickelt, die auf das Leben in einem anderen politischen System mit völlig anderen wirtschaftlichen, rechtlichen und sozialen Rahmenbedingungen ausgerichtet und dafür mehr oder weniger gut angepaßt waren. Nun haben sich diese Rahmenbedingungen vollständig geändert. Damit entsteht die hier zu behandelnde Frage, welche Bedeutung diese Umbrüche für die Menschen haben, und wie sie die erforderlichen Umstellungen bewältigen.

Es kann nicht erwartet werden, daß die handelnden Individuen mit dem abrupten Systemwechsel auch alle damit verbundenen Ziele austauschen und sofort die entsprechenden Verhaltensweisen übernehmen sowie die für das Funktionieren des neuen Systems erforderlichen Kompetenzen bereit halten. Zunächst ist daher zu fragen, mit welchen Handlungsorientierungen die Menschen im System der ehemaligen DDR aufgewachsen sind; ob und wieweit diese mit den gegenwärtigen Handlungserfordernissen und -bedingungen kompatibel sind; wie die Menschen die jetzigen Handlungsbedingungen bewerten; ob sie sie übernehmen wollen; welche Voraussetzungen sie dafür mitbringen, und welche psychologischen Probleme bei der Übernahme entstehen. Dies wird sich auf die weiteren Transformationsprozesse auswirken, jedenfalls soweit individuelles Handeln die Funktionsfähigkeit von Institutionen und des sozio-politischen Systems beeinflußt.

Wie die Menschen in den neuen aber auch in den alten Bundesländem den Transformationsprozeß erleben, wie sie von ihm beeinflußt werden und ihn ihrerseits beeinflussen - diese Wechselwirkungsprozesse sind Teil des allgemeinen Verhältnisses von Individuum und Gesellschaft. Zur Analyse der Transformationsprozesse ist daher also auch eine psychologische Betrachtungsweise erforderlich. Um Art und Verlauf des Transformationsprozesses genauer zu beschreiben und ggfs. zu erklären, müßten also die Beziehungen zwischen der Individual-, der Mikro- und der Makroebene im soziopolitischen Wandel genauer geklärt werden.

Im folgenden müssen wir uns jedoch darauf beschränken, aus der Vielzahl von Faktoren, die die Bewältigungsprozesse beeinflussen können, aus übergeordneten Ge- 
sichtspunkten einige psychologische Erklärungskonzepte zu diskutieren. Diese sollen Wechselwirkungen zwischen individuellen und gesellschaftlichen Bedingungen beleuchten. Zunächst werden einige psychologische Ausgangsbedingungen, die durch die bisherige Sozialisation, den Systemwechsel und die folgenden Transformationsprozesse entstanden sind, behandelt. Dies soll klären, was die Umbruchssituation für Ostdeutsche subjektiv bedeutet und welche Anforderungen bestehen. Dann sollen mögliche psychologische Probleme diskutiert werden. Bei unserer Analyse werden wir uns auf ausgewählte Schnittstellen zwischen sozialem Wandel und individuellen Prozessen, wie Handlungsorientierungen, System- und Selbstbewertungen und Bewältigungsstrategien der Kontrollorientierung konzentrieren. Am Schluß werden einige methodische Probleme bei der Untersuchung psychologischer Aspekte im Transformationsprozeß erörtert.

\subsection{Psychologische Prozesse bei Umbrüchen im "Lebensraum"}

\subsubsection{Umbrüche im "Lebensraum" und Anforderungen an den Einzelnen}

Der enorme historische Umwälzungsprozeß mit dem grundlegenden sozio-ökonomischen und politischen Wandel hat zu massiven Umbrüchen im "Lebensraum" (Lewin, 1935) des Einzelnen geführt. Im folgenden wird gefragt, wie die Änderungen bisheriger Lebensbedingungen und des individuellen Handlungsfeldes vom Einzelnen wahrgenommen, erlebt und verarbeitet werden und welche individuellen Unterschiede darin bestehen. Durch die Transformation werden die Menschen vor neue Rahmenbedingungen gestellt, die ihre bisherigen Handlungsoptionen plötzlich erweitern aber auch einschränken. Neue Regeln und Ordnungssysteme wirken sich auf Entscheidungen bis in den Alltag hinein aus. Die Menschen der neuen Bundesländer haben ein neues System übernommen und stehen vor der Aufgabe, an dessen Funktionieren mitzuwirken. Das neue System erfordert Umstellungen und Anpassungen, die Verluste aber auch neue Chancen bedeuten können. Die politischen und wirtschaftlichen Umbrïche, die sozialstrukturelle und regionale Mobilität, die neue Rechtslage dies führt zu massiven sozialen und psychischen Herausforderungen.

Die im bisherigen System aufgebauten Handlungsorientierungen treffen nun auf neue soziale, politische und wirtschaftliche Bedingungen. Dies führt mindestens zu ungewohnten und häufigen Diskrepanzerfahrungen, Unsicherheiten und Ambivalenzen, die $\mathrm{zu}$ verarbeiten sind. Einerseits vermittelt das neue Wirtschaftssystem erwünschte neue Konsummöglichkeiten, andererseits bringt es die Gefährdung des Arbeitsplatzes mit sich. Das politische System vermittelt neue Partizipationsformen aber auch unübersichtliche Bürokratisierungen. Es besteht Unsicherheit, ob es sich lohnt, neue Anstrengungen (z.B. für Umschulung) auf sich zu nehmen. Diese Unsicherheiten hängen mit den unklaren Handlungsoptionen, dem schnellen Wandel von Optionen und dem unbestimmten Ausgang von Handlungsergebnissen bzw. den eigenen Kontrollmöglichkeiten zusammen. Für manche Personen bedeuten diese erhebliche Belastungen, die Fehlanpassungen und anomische Reaktionen zur Folge haben; für andere bedeuten sie Handlungschancen, die aktiv und konstruktiv gestaltet werden können. Mit der Umstrukturierung ihres Lebensraumes akkumulieren sich für einige die Belastungen ( $z, B$. bei Frauen; wenn mehr als ein Familienmitglied arbeitslos ist; oder 
wenn mit der Scheidung der Verlust des Arbeitsplatzes zusammenfällt); für andere entstehen plötzlich erfolgversprechende neue Chancen.

Die objektiven Umbrüche haben bereits massive Verhaltensänderungen ausgelöst, die jedoch keineswegs schon deswegen gelungene psychologische Verarbeitungen bedeuten müssen; vielmehr haben sie ihrerseits zu geänderten Ausgangsbedingungen im Transformationsproze $\beta$ geführt, die wiederum vom Einzelnen psychologisch zu verarbeiten sind. Solche Änderungen zeigen sich u.a. darin, daß etwa $50 \%$ der ostdeutschen Bevölkerung 1990/91 die Beschäftigung gewechselt haben; mehr als $60 \%$ der Ende 1990 in der Industrie beschäftigten Arbeitnehmer wurden entlassen; ca. $40 \%$ der Bevölkerung sind direkt oder indirekt (z.B. über einen Familienangehörigen) von Arbeitslosigkeit betroffen; die Abwandenung aus den neuen Ländern nimmt durch die Erhöhung der Arbeitslosigkeit (trotz Anpassung der Löhne in Ost und West) zu.

\subsubsection{Annahmen über psychologische Faktoren bei der Bewäligung von Umbrüchen}

Die Anforderungen an Ostdeutsche durch den Systemwechsel und die Transformationsprozesse sind beträchtlich. Die individuelle Verarbeitung des geänderten Lebensraumes hängt von einer Vielzahl von Bedingungen $a b$, die interindividuell unterschiedlich sind. Wir gehen hier von einem handlungstheoretischen Ansatz aus, der Annahmen verschiedener theoretischer Ansätze verwendet, da es keine umfassende psychologische Theorie der Transformationsprozesse gibt. Psychologische Bedingungen für einen erfolgreichen Umgang mit den einschneidenden Änderungen im Lebensraum lassen sich aus einigen persönlichkeits-, sozial- und entwicklungspsychologischen Theorien ableiten, auch wenn diese nicht für solche Fragen entwickelt wurden. Wir greifen hier u.a. auf die Persönlichkeitstheorie von Lewin (1935), auf Motivationstheorien (Heckhausen, 1989), kognitive Theorien wie die Selbsttheorie von Epstein (1980), die Dissonanztheorie von Festinger (1957), auf Gerechtigkeitstheorien (Bierhoff, Cohen \& Greenberg, 1986), Theorien zu Kontrollüberzeugung (Rothbaum, Weisz \& Snyder, 1982), und Theorien zum "Coping" bei kritischen Lebensereignissen und zur Bewältigung von Stress (Lazarus \& Folkman, 1984) zurück.

Wir nehmen im Sinne von Lazarus und Folkman (1984) an, daß zunächst die neue Lage bewertet wird. Im Sinne von Lewin (1935) nehmen wir weiter an, daß diese Bewertungen und die dadurch bedingten Handlungsziele von den bisherigen Erfahrungen abhängen. Bei der Analyse intrapsychischer Verarbeitungsprozesse können wir jedoch nicht stehen bleiben. Vielmehr sind die Auswirkungen auf das Verhalten zu untersuchen. Da wir davon ausgehen, daß Verhalten in Interaktionskontexten erfolgt, nehmen wir weiter im Sinne von Lewin an, daß das soziale Umfeld die Art der Bewältigung der Anforderungen beeinflußt. Je nach den in der Sozialisation aufgebauten Handlungsorientierungen - einschließlich Selbstkonzept und Kompetenzen - und je nach gegebenem sozialen Netz- und Bezugssystem müßten also die Herausforderungen des Wandels bewertet und bewältigt werden.

Entsprechend müßte zunächst gefragt werden, wie die psychologischen Ausgangsbedingungen aussehen. Dann ist $\mathrm{zu}$ fragen, wie die vergangenen und gegenwärtigen Lebenslagen subjektiv bewertet werden. Wird z.B. die gegenwärtige eigene Lebenslage (im Vergleich zu früher und/oder im Vergleich $\mathrm{zu}$ anderen) als besser oder schlechter, als Ergebnis gerechter oder ungerechter Verteilung, als Gewinn oder per- 
sönlicher Mißerfolg, eher als Ergebnis eigener Fehler oder eher als Versagen externer, z.B. politischer Kräfte bewertet? Dann müßte nach den intrapsychischen Folgen dieser Bewertungen gefragt werden. Haben solche Bewertungen Folgen für das Selbstkonzept (Identität), und führen sie zur internen Reorganisation von Handlungsorientierungen, die der Anpassung an neue Rahmenbedingungen mehr oder weniger gut dienen? Werden bisherige Ziele und Kompetenzen in die neuen Anforderungsbedingungen integriert und neue Handlungsorientierungen aufgebaut, um neue Optionen zu nutzen und Mißerfolge und Risiken zu vermeiden? Dazu sind bestimmte Überzeugungen und Deutungsmuster notwendig, die wir hier als Kontroll- und Zukunftsorientierung verstehen und auf die später eingegangen werden soll. Gemäß solchen Kontrollund Zukunftsorientierungen werden vorgegebene neue Anforderungen eher als Chancen und weniger als selbstwertbelastende Frustration und Bedrohung gedeutet; neue Kompetenzen werden aufgebaut und bisherige Ziele werden so modifiziert, daB eine erfolgsmotivierte Handlungsplanung erfolgen kann, bei der Risiken, eigene Anstrengungen und Verzichtleistungen akzeptiert werden.

SchlieBlich stellt sich die Frage, wie sich solche intrapsychischen Prozesse auf Verhalten auswirken, also z.B. auf soziale Anpassung oder Gewalt, auf die Berufswahl, die Art der politischen Beteiligung, auf Migrationsentscheidungen - also Verhalten, das wiederum sozio-politische Folgen hat. Wie die Herausforderungen bewältigt und verhaltenswirksam werden, hängt also vermutlich u.a. davon ab, wie der Einzelne die neue Lage bewertet und welche Handlungsorientierungen er mitbringt. Dies wiederum hängt davon ab, wie der Einzelne aufgewachsen ist, und in welchem sozialen Netzwerk und Bezugssystem er eingebunden ist.

Im nächsten Schritt einer Wechselwirkungsanalyse müßten dann die intrapsychischen und sozialen Folgen dieses Verhaltens auf den Verlauf der Transformationsprozesse studiert werden. Hier sind weiterführende theoretische Ansätze erforderlich, die über die Analyse aktueller Prozesse der Anpassung an Umbrüche hinaus auch die allgemeinen Beziehungen zwischen Individuum und Gesellschaft und diese im Prozeß des Wandels untersuchen. Dies kann hier jedoch nicht geleistet werden.

Im folgenden Abschnitt sollen ausgewählte psychologische Aspekte des Transformationsprozesses diskutiert werden, die sich einerseits aus den Anforderungen für individuelles Handeln und andererseits aus den möglichen Folgen dieses Handelns (für die Transformation) ergeben. Dazu wird zunächst gefragt, welches die psychologischen Ausgangsbedingungen für Ostdeutsche waren und sind.

\section{Schnittstellen von psychologischen und sozialen Faktoren}

\section{I Ausgangsbedingungen für Ostdeutsche: Vorüberlegungen}

Die "Ausgangsbedingungen" für Ostdeutsche müßten sich aus einer Bestandsaufnahme der Bedingungen vor und nach der "Wende" sowie im Vergleich zu Westdeutschen ergeben. Daß sich diese "Ausgangsbedingungen" heute wohl kaum "objektiv" beschreiben lassen, ist u.a. auch ein psychologisches Problem und wohl ein Effekt bisheriger Transformationen. Im folgenden wurden als "Ausgangsbedingungen" einige Sozialisationsaspekte sowie einige gesellschaftlich relevante individuelle Orientierungen wie Werthaltungen, System- und Selbstbewertung skizziert, die jeweils Schnitt- 
stellen zwischen Individuum und Gesellschaft sind. Zunächst soll jedoch nach Ähnlichkeiten und Unterschieden zwichen Ost- und Westdeutschen gefragt werden. Kulturhistorische Argumente sprechen dafür, davon auszugehen, daß Ost- und Westdeutsche sich nicht wesentlich voneinander unterscheiden. Es sind Deutsche mit der gleichen Sprache und bis Ende der vierziger Jahre auch mit der gleichen Kultur und Geschichte.

Auch aufgrund modernisierungstheoretischer Ansätze wird angenommen, daß heute eher Ähnlichkeiten als Unterschiede zwischen beiden deutschen Staaten bestehen, weil sich in allen hochindustrialisierten Ländern (und die DDR gehörte dazu), unabhängig von jeweiligen Ideologien, gleiche Lebensstile wie "pluralisierte" Lebensformen (z.B. in der Familie) vollzögen. Hier werden jedoch relativ vereinfachte Annahmen zugrunde gelegt, z.B. daß Prozesse des Wandels in hochindustrialisierten Gesellschaften sowie deren Wirkungen (z.B. "postmoderne" Werthaltungen, Pluralisierung und Individualisierung von Lebensformen) gleichförmig seien. "Kulturspezifische" Modernisierungsformen (Bendix, 1965/66) und damit zusammenhängende Werthaltungen (vgl. Trommsdorff, 1989b) sowie vor allem die Bedeutung solcher Werthaltungen für verschiedene Etappen in der Persönlichkeitsentwicklung werden dabei unzureichend berücksichtigt.

Aufgrund sozialisationstheoretischer Annahmen sind differenziertere Hypothesen über Ähnlichkeiten und Unterschiede abzuleiten. Bis Ende der vierziger Jahre bestanden in Ost- und Westdeutschland etwa gleiche Lebensverhältnisse und ein relativ ungestörter Austausch zwischen beiden Teilen Deutschlands. Dann wuchs die Grenze, und die beiden deutschen Staaten entwickelten sich in unterschiedlicher Richtung. Die unterschiedlichen politischen, wirtschaftlichen und sozialen Systeme beeinflußten die Sozialisation, den Alltag und damit schließlich auch das Denken und Handeln der Bevölkerung in beiden Teilen Deutschlands. Dies dürfte sich kohortenspezifisch unterschiedlich ausgewirkt haben (vgl. Mayer, 1993). Ostdeutsche, die Anfang der 40er Jahre geboren sind, und die in der Wendezeit etwa 45 Jahre alt waren, teilen mit Westdeutschen dieser Generation viele Gemeinsamkeiten, insbesondere aus der frühen und mittleren Kindheit, die in die Kriegs- und Nachkriegszeit fiel. Die danach Geborenen sind jedoch von Anfang an in verschiedenen Systemen sozialisiert worden und dürften ganz unterschiedliche Entwicklungen durchlebt haben. Die jüngere Generation hat in den 80er Jahren eine gewisse Aufweichung des Systems erlebt und Kenntnis über den Westen gewonnen, was wieder auf Ähnlichkeiten in Werthaltungen bei ostund westdeutschen Jugendlichen schließen läßt.

Diese Annahmen über Ähnlichkeiten in der "Mentalität" und Kultur beider Teile Deutschlands beziehen sich auf Prozesse vor der Wende und müßten durch empirische Daten aus dieser Zeit geprüft werden. Daten zum Vergleich von Gemeinsamkeiten und Unterschieden, die nur nach der Wende erhoben wurden, sind für die Prüfung dieser Annahmen unzureichend und unter Gefahr von Voreingenommenheiten interpretierbar. Nach der Wende festgestellte Gemeinsamkeiten können sowohl als Beleg für das gemeinsame kulturelle Erbe, als Beleg für die vor der Wende erfolgten gleichen Modernisienungsprozesse oder als Beleg für die im Laufe des Transformationsprozesses geleisteten Anpassungen an westliche Bedingungen gedeutet werden. Entsprechend lassen sich Unterschiede nach der Wende als Beleg für die eigenständige Entwicklung in beiden Teilen Deutschlands, als erfolgreiche Abwehr westlicher Ein- 
flüsse oder als Beleg für die persönlichkeitsbildende Wirkung des DDR-Regimes interpretieren.

Es ist jedoch zunächst schwierig, überhaupt festzustellen, zu welchem Zeitpunkt welche Ähnlichkeiten und Unterschiede in der west- und ostdeutschen Bevölkerung bestanden haben; ob sich diese eher auf soziale oder auf psychologische Merkmale beziehen; an welchen Kriterien diese als Ähnlichkeiten oder Unterschiede gemessen und gedeutet werden können; ob diese stabil bleiben oder sich ändern; worauf diese beruhen, und welche Wirkungen sie schließlich haben. Eine unvoreingenommene Deutung der Befunde, die frei von impliziten Bewertungen ist, scheint schwierig zu sein. Es ist auch nicht auszuschließen, daß ost- und westdeutsche Sozialwissenschaftler von einer unterschiedlichen Perspektive ausgehen, die von Vorstellungen über "wünschenswerte" Gemeinsamkeiten und Unterschiede im Ost-West-Vergleich geleitet sind. Ob Ähnlichkeiten oder Unterschiede einen Nachteil für die innere Einigung beider Teile Deutschlands oder für eine erfolgreiche Anpassung Ostdeutscher an die Transformationsprozesse bedeuten, wäre eine weitere Frage, die wir hier nicht klären können.

\subsection{Sozialisationsbedingungen}

Die individuelle Sozialisation und die dort aufgebauten Werthaltungen, Handlungsorientierungen sowie auch Investitionen stellen die eigentlichen Ausgangsbedingungen für die subjektive Bewertung der Umbrüche und für die individuellen Verarbeitungsleistungen dar.

Allgemeine Sozialisationserfahrungen. Typisch für die Sozialisation in Ostdeutschland war eine Erziehung in einem totalitären politischen System, das von der übrigen Welt, insbesondere vom Westen und seiner pluralistischen Gesellschaft abgeschlossen war. In Ostdeutschland bestand anders als im Westen eine uniformistische Gesellschaftsform mit einheitlicher Ideologie und vereinheitlichten Lebensformen; diese Gesellschaft kontrollierte den Einzelnen, aber sie behütete ihn auch. Die wirtschaftlich begrenzten Verhältnisse ohne Privateigentum ließen wenig individuelle Verantwortung und Eigeninitiative zu, es sei denn, solche Aktivitäten standen im Dienst an der Partei und waren mit hohen Anpassungsleistungen verbunden. Dazu kommen die spezifischen Sozialisationsbedingungen. Diese beinhalteten über die hier skizzierten Rahmenbedingungen hinaus besondere Formen der in der frühen Kindheit einsetzenden Kinderkrippenerziehung (mit ihren Einflüssen auf die Persönlichkeitsentwicklung) und des einheitlichen Schulsystems. Der Erziehung lag ein "tabula rasa"-Modell und Gleichheitsaxiom zugrunde, das jedoch in der Praxis scheiterte (Schmidt, 1992; Trommsdorff, 1992).

Kohorten- und entwicklungsspezifische Probleme. Je nachdem in welcher Kohorte man aufgewachsen ist und welche Einstellungen, Gewohnheiten und Ressourcen dort aufgebaut wurden, bestehen unterschiedliche Ausgangsbedingungen für die Verarbeitung des Wandels (Elder, 1979). Es ist weiter zu erwarten, daß Verhaltensoptionen, Verluste und Chancen kohortenspezifisch verschieden sind. Zwei Drittel der jetzt in den östlichen Bundesländern lebenden Deutschen sind nach 1949 geboren und im System 
der DDR aufgewachsen. Es wird einen Unterschied in den Bedingungen für die Verarbeitung des Wandels bedeuten, ob man nach 1950 oder vor dem Zweiten Weltkrieg sozialisiert wurde, d.h. unter welchen jeweilig vorherrschenden Denkmustern, wirtschaftlichen und politischen Bedingungen (die periodisch unterschiedlich starke Anpassungszwänge vermittelt haben) man aufgewachsen ist.

$\mathrm{Zu}$ fragen ist daher zum einen im Sinne von Huinink und Mayer (1993) und Mayer (1993), unter welchen sozialen und psychologischen Bedingungen verschiedene Kohorten in der ehemaligen DDR gelebt haben und vor allem, mit welchen daraus erwachsenen Einstellungen und Erwartungen diese den Übergang in ein völlig anderes System vollziehen. Zum anderen ist im Sinne von Baltes und Baltes $(1989 ; 1990)$ danach zu fragen, welche Ressourcen die jeweiligen Altersgruppen aufgebaut haben, und zwar in bezug auf die jeweils gegebenen Optionen, Erwartungen und Lebensbedingungen im System der ehemaligen DDR; und was von diesen selektiven Investitionen nach den Umbrüchen jetzt noch funktionell ist, was daran die Verarbeitung des Wandels fördert, erschwert oder gar behindert. Für die älteren Kohorten bestehen erschwerte Bedingungen, z.B. neue Kompetenzen für eine Verarbeitung der Umbrüche aufzubauen. Die beruflichen Optionen sind für ältere Kohorten gering. Die über 40-Jährigen müßten sich wegen geänderter Arbeitsmarktbedingungen eigentlich beruflich neu orientieren; geringe Chancen, einen Arbeitsplatz zu finden, lassen dies jedoch als eine sinnlose Investition erscheinen ("verlorene Generation").

Darüber hinaus sind die Anpassungsprozesse an die Transformation generell für solche Personen erschwert, die über einen Rollenwechsel hinaus aufgrund von Statuspassagen (z.B. Frührentner) erhebliche interne Umorientierungen zu vollziehen haben. Eine Akkumulation von Belastungen hängt nicht nur von der Kohortenzugehörigkeit und damit verbundenen Ausgangsbedingungen, sondern auch vom Entwicklungsalter $\mathrm{ab}$.

So durchlaufen ostdeutsche Jugendliche massive Rollenwechsel. Einerseits scheinen Jugendliche gute Voraussetzungen für die Verarbeitung der Umbrüche mitzubringen, da sie sich in den Jahren vor der Wende bereits mit westlichem Denken vertraut machen konnten. Sie haben noch kaum vergebliche Investitionen in Bildung und Beruf zu beklagen. Andererseits ist bei ihnen die Entwicklung einer eigenen Identität noch nicht abgeschlossen. Darüber hinaus stehen sie vor besonderen Entwicklungsaufgaben: so ist der entwicklungsbedingte Konflikt zwischen Autonomie und Bindung in Auseinandersetzung mit den Eltern und der Altersgruppe zu lösen. Dazu kommen die transformationsbedingten Umstellungen (u.a. Verlust von sozialen Netzen). Berufswechsel oder Verlust des Arbeitsplatzes der Eltern verstärken Belastungen von Jugendlichen, wie Längsschnittstudien in den USA zeigen (Flanagan \& Eccles, 1993). Bei den gegebenen Anforderungsbedingungen und bei der bisher erfahrenen Sozialisation dürften daher für Jugendliche die Umstellungen erheblich sein.

Sozialisation und Transformationsprozesse. Diese besonderen Sozialisationsbedingungen begründen Annahmen über beträchtliche Unterschiede in Handlungsorientierung, Lebenszielen, Erwartungen und Verhaltensmustern bei Ost- und Westdeutschen. Diese sozialisationsbedingten Unterschiede müßten nach dem Beitritt besondere Umstellungsprobleme und Anpassungsleistungen für Ostdeutsche im TransformationsprozeB bedingen. Dies ist allerdings je nach Kohortenzugehörigkeit und Entwicklungsalter zu differenzieren (vgl. Schmidt \& Heckhausen, in diesem Band). 
Umgekehrt wirken sich die Transformationsprozesse u.a. durch die geänderten Sozialisationsinstitutionen sowohl auf Eltern und Lehrer als auch auf Kinder und Jugendliche sowie auch auf deren Beziehung zueinander und zu den neuen Institutionen aus. Zwar sind Erzieher und Erzogene jeweils unterschiedlichen Anpassungsanforderungen an die Transformation ausgesetzt; sie werden mit jeweils verschiedenen Problemlagen konfrontiert und verfügen über unterschiedliche Ressourcen zu deren Bewältigung. Jedoch stehen beide aus interaktions- und handlungstheoretischer Sicht in wechselseitiger Beziehung zueinander (vgl. Hurrelmann, 1986). Diese Konstellation ist Teil und Ergebnis von Sozialisationsprozessen und müBte als Ausgangsbedingung für die Wirkung der Transformationsprozesse auf den Einzelnen in dessen Verhältnis zur Gesellschaft analysiert werden. Daher ist für die Analyse der Transformationsprozesse und damit verbundenen Fragen nach den Beziehungen zwischen Individuum und Gesellschaft im Wandel das Studium von Sozialisationsbedingungen von besonderer Bedeutung. Insbesondere aus sozialisationstheoretischer Sicht lassen sich die wechselseitigen Zusammenhänge zwischen gesellschaftlichen, wirtschaftlichen und politischen Transformationsprozessen und individuellem Handeln erfassen.

\subsection{Werthaltungen in Ost- und Westdeutschland}

Im folgenden sollen Ähnlichkeiten und Unterschiede in Werthaltungen diskutiert werden, da Werthaltungen teilweise Aufschlüsse über psychologische Ausgangsbedingungen für Anpassungen an die Transformationsprozesse geben. Die Reanalysen von Werthaltungen vor der Wende in beiden Teilen Deutschlands zum Ende der 80er Jahre von Gensicke (1992) weisen auf hohe Ähnlichkeiten hin, die als eine Abkehr von kollektivistischen Werten des SED-Regimes in der Bevölkerung der ehemaligen DDR und eine Orientierung auf individualistische Werte wie Selbstbestimmung, Konsumorientierung und Umweltbewußtsein interpretiert werden. Auch Friedrich und Griese (1991) belegen für ost- und westdeutsche Jugendliche für die Zeit vor der Wende eine hohe Übereinstimmung von Werten. Die Autoren deuten dies als Ergebnis eines auffälligen Wertewandels ostdeutscher Jugendlicher seit 1975 mit einer Hinwendung zu individualistischen, materialistisch-hedonistischen Werten.

Die Autoren führen diesen Wandel auf zunehmenden Kontakt mit westlichen Medien zurück, wodurch Jugendliche über westliche Lebensgewohnheiten informiert gewesen seien; durch ihre geringere Einbindung in das SED-Regime und Nutzung der Möglichkeiten einer "Nischen-Gesellschaft" hätten sie sich stärker in Richtung "postmoderner" Werte hin orientieren und einen "heimlichen" Wertewandel vollziehen können. Nach dieser Deutung mußte man sich zwar in öffentlichen Institutionen den Vorgaben des SED-Regimes anpassen, konnte sich jedoch in der Familie und im Freundeskreis einen weitgehend vom öffentlichen Bereich abgekoppelten privaten Bereich, in dem eigene Werthaltungen formiert wurden, schaffen. Zwar mag in den 80er Jahren eine Ausgrenzung aus dem totalitären System für Jugendliche leichter als für ihre Eltern gewesen sein; jedoch hat der Wertewandel nach den Analysen von Gensicke (1992) weite Teile der Bevölkerung mit erfaßt. Auch in Familienformen und Lebensstilen wurden in den 80er Jahren in beiden Teilen Deutschlands ähnliche "pluralistische" Werthaltungen demonstriert. Allerdings ist zu fragen, ob tatsächlich 


\section{"postmoderne" Werte und "pluralistische" Lebensformen in der ehemaligen DDR dasselbe wie im Westen bedeutet haben.}

Ähnliche Werthaltungen in Ost- und Westdeutschland vor der Wende und deren Diskrepanz zur SED-Ideologie bzw. die Wahmehmung ostdeutscher Bürger, daß die westlichen Werte und die entsprechenden Ziele und Hoffnungen im DDR-Regime nicht zu befriedigen sein würden, werden von manchen Autoren als eine der Ursachen für den Systemumbruch gedeutet. Nach dieser Sicht dürften Ähnlichkeiten in Werthaltungen in den alten und neuen Bundesländem direkt nach der Wende keineswegs als ein Ergebnis der Transformationsprozesse, sondern vielmehr als eine Voraussetzung für die Wende und für den folgenden Systemumbruch gedeutet werden. Für unsere Frage nach den psychologischen Ausgangsbedingungen für die Transformationsprozesse ist jedenfalls von Bedeutung, daß empirische Daten eine relativ hohe Übereinstimmung von Werthaltungen bei Jugendlichen der ehemaligen DDR und der Bundesrepublik vor und nach der Wende nahelegen.

So geht aus vorliegenden Umfragestudien nach der Wende hervor, daß hohe Ähnlichkeiten von ost- und westdeutschen Jugendlichen bestehen, die zunächst nicht ohne weiteres auf besondere Anpassungsprobleme ostdeutscher Jugendlicher schließen lassen. Repräsentative Jugendsurveys aus der Zeit nach der Wende an hinreichend großen Stichproben belegen auf den ersten Blick hohe Ähnlichkeiten von individualistischen Werthaltungen und Lebenszielen (Berufs- und Bildungsziele) bei Jugendlichen der alten und neuen Bundesländer (vgl. die "Schülerstudie 90" von Behnken et al., 1991; die Befragungen des DJI von Dennhardt et al, 1990, bzw. die Schülerstudie des DJI, 1992; oder der über den deutsch-deutschen Vergleich hinausgehende Deutsch-Polnische Jugendreport von Melzer, Lubowski \& Schmidt, 1991).

Allerdings zeigen sich deutliche Geschlechts- und Bildungsunterschiede. Mädchen im Vergleich zu Jungen verfolgen z.B. weniger ausgeprägte materialistische Lebensziele (guter Verdienst) und zwar besonders in Ostdeutschland (vgl. Gille, 1992). Deutliche Unterschiede bestehen zwischen west- im Vergleich zu ostdeutschen Jugendlichen auch je nach Zugehörigkeit zu verschiedenen Bildungsgruppen. So wird z.B. die Vereinigungspolitik besonders von den höheren Bildungsgruppen westdeutscher und den unteren Bildungsgruppen ostdeutscher Jugendlicher eher befürwortet, bzw. von den ostdeutschen Jugendlichen mit höherem und westdeutschen Jugendlichen mit niedrigem Bildungsgrad eher abgelehnt (Behnken et al., 1991). In diesen Einstellungen spiegeln sich vermutlich die erwarteten Berufschancen und Anpassungsprobleme, die für die jeweiligen Bildungsgruppen in den alten im Vergleich zu den neuen Bundesländem unterschiedlich sind.

Darüber hinaus bestehen deutliche Kohortenunterschiede in bezug auf Wertvorstellungen. Ostdeutsche Jugendliche unterscheiden sich nach diesen Studien erheblich von der Eltem\#generation. Generationsunterschiede belegt auch Gensicke (1992) in einer von der Kommission für die Erforschung des sozialen und politischen Wandels in den neuen Bundesländern e.V. (KSPW) geförderten Kurzstudie. Danach bestehen zwischen den Altersgruppen erhebliche Unterschiede in den Einstellungen zur gesellschaftlichen und politischen Partizipation, zur Arbeit und zu Lebenszielen. Die jüngeren Befragten sind engagierter und vertreten stärker individualistische, hedonistische und materielle Werte; gleichzeitig sind sie weniger informiert und weniger politisch interessiert als die über 40-Jährigen. Die in der Elterngeneration in Ost- und Westdeutschland bestehenden unterschiedlichen Werthaltungen deuten auf generationsspe- 
zifisch unterschiedliche Ausgangsbedingungen und Anpassungsanforderungen an Transformationsprozesse hin, die durch die Sozialisationserfahrungen der jeweiligen Altersgruppen bedingt sind.

\subsection{Systembewertung und Systemidentifikation}

Subjektive Bewertung der beiden Systeme als psychologische Ausgangsbedingung. Werthaltungen sind individuelle Orientierungen, die den Verlauf institutioneller Transformationen und die individuelle Anpassung an den Wandel beeinflussen. Teil solcher individueller Orientierungen sind die Bewertung der Systeme der ehemaligen DDR und der Bundesrepublik Deutschland und ihrer Institutionen, Parteien und Verbände. Diese Bewertungen stellen daher ihrerseits eine Voraussetzung für die individuelle Verarbeitung des Systemumbruchs dar. Aspekte von Systembewertungen sind System- und Parteienidentifikation, nationale Identität, Gerechtigkeitsvorstellungen und politische Partizipationsformen. Damit sind dies psychologisch relevante Ausgangsbedingungen für Anpassungen im Transformationsprozeß. Es ist zu vermuten, daß sich die subjektive Bewertung der ehemaligen DDR und der Bundesrepublik und die Erwartungen an diese Systeme auf die Erfüllung individueller Bedürfnisse im Verlaufe der Transformationsprozesse geändert haben.

Ambivalenz von Bewertungen. Mit dem politischen, wirtschaftlichen und sozialen $\mathrm{Zu}-$ sammenbruch der DDR wurde für die Menschen in Ostdeutschland ein System zerstört, das einerseits ihre individuelle Freiheit, Freizügigkeit, soziale Mobilität oder politische Partizipation massiv eingeschränkt hat, das ihnen andererseits auch relative wirtschaftliche und soziale Sicherheit und eindeutige Handlungsregeln geboten hat. Die Bedeutung des Zusammenbruchs der ehemaligen DDR und die Übernahme des Systems der Bundesrepublik dürfte für viele sehr ambivalent sein und individuell verschieden bewertet werden - für einige als Gewinn und für andere als Verlust. Entsprechend kann das neue System der Bundesrepublik psychologisch ganz Verschiedenes bedeuten. Der Zuwachs an Freiheit und Selbstbestimmung ist mit einer Vielzahl von Handlungsoptionen verbunden; auch diese können einerseits als Verunsicherungen und Verlust von individueller Kontrolle und andererseits als Förderung von individuellen Chancen erlebt werden. Das demokratische System mit seinen vielfältigen Verwaltungsstrukturen kann einerseits als unübersichtlich und einengend und andererseits als vorhersagbar und als individueller Schutz erlebt werden. Anforderungen an individuelle Leistung und Initiative in dem marktwirtschaftlichen System können als Förderung von Egoismus und als Widerspruch zu hoch geschätzten Solidaritätswerten, sie können auch als Chance für die Erprobung eigener Fähigkeiten und der Verbesserung der eigenen (wirtschaftlichen) Unabhängigkeit erlebt werden. Der Zusammenbruch institutionalisierter sozialer Netze, wie sie beispielsweise durch die sozialistischen Jugendorganisationen bestanden, kann ein Verlust von Sicherheit, Geborgenheit und bewährter Einbindung in eine soziale Gruppe und damit eine Belastung und ein Risiko für den Einzelnen bedeuten. Dieser Institutionenwandel kann aber auch als Befreiung von Zwang und als Chance für die Wahl eigener sozialer Bezugsgruppen erlebt und genutzt werden. Ähnlich kann das marktwirtschaftliche System mit drohendem Verlust des Arbeitsplatzes als Belastung oder auch als Chance 
für Weiterbildungs- und Umschulungsmaßnahmen im Sinne einer Erweiterung von Kompetenzen und einer Anpassung an geänderte Anforderungen des Arbeitsmarktes und der Wirtschaft verstanden und genutzt werden. So sind widersprüchliche und ambivalente Bewertungen wahrscheinlich. Diese dürften erhebliche Verunsicherungen bewirken.

Erwartungen. Bewertungen beziehen sich nicht nur auf die vergangenen und gegenwärtigen, sondern auch auf die zukünftigen, erwarteten Systembedingungen. Bei einigen Menschen bestehen eher positive Erwartungen an das übernommene System und Hoffnungen auf endlich erfüllbare Wünsche nach Eigentum, Freiheit und Selbstbestimmung, während bei anderen eher Befürchtungen und Sorgen bestehen, in dem neuen System überhaupt zurechtzukommen. Die positiven und die negativen Erwartungen stellen wiederum jeweils unterschiedliche psychologische Ausgangsbedingungen für den Transformationsprozeß dar, da sie jeweils auch unterschiedliche Anpassungsprobleme und Bewältigungsformen implizieren. Von Art und Ausmaß der Hoffnungen hängt das Ausmaß der Enttäuschungen $a b$, wenn nach der Wende die Wünsche nicht erfüllt wurden. Je nachdem ob die Wende und der Beitritt erhofft oder befürchtet wurden, und je nachdem mit welchen Hoffnungen und Befürchtungen die mit der Wende einsetzenden Transformationsprozesse erlebt wurden, dürfte die Anpassungsbereitschaft an die Transformation und die Frustrationstoleranz bei Erwartungsenttäuschungen unterschiedlich sein.

Systemidentifikation und Identität. Darüber hinaus werden Unterschiede in der Bewältigung von Transformationsproblemen auch darin bestehen, ob und wie weit sich Ostdeutsche mit dem System der ehemaligen DDR identifiziert haben und sich mit diesem System heute noch eng verbunden fühlen. Menschen, für die die Tätigkeit in diesem System der eigentliche Lebensinhalt war, dürften den Zusammenbruch dieses Staates, mit dem man sich identifiziert und demgegenüber man sich loyal verhalten hat, auch wenn man vielleicht manches an dem Regime abgelehnt hat, als ein ernstes Identitätsproblem erleben. Das muß nicht nur für Parteifunktionäre, Lehrer, NVAAngehörige oder die Polizei gelten. Dieses Identitätsproblem wird durch andere Beeinträchtigungen (wie Bedrohung des Arbeitsplatzes, der sicheren sozialen Einbettung, der eigenen Funktionsfähigkeit und sozialen Bedeutung), die in jedem Fall belastend sind, gleich ob sie Ost- oder Westdeutsche treffen, zusätzlich erschwert. Ein spezifischer Aspekt der Identitätsproblematik für Ostdeutsche besteht jedoch darin, daß die Identifikation mit dem System der DDR durch den Zusammenbruch des Systems zu einem Zusammenbruch der eigenen Welt- und Lebensdeutung führen kann. Darüber hinaus bedeutet dieser Zusammenbruch den Verlust der eigenen eigentlich relevanten Bezugsgruppe, deren Funktion ja darin besteht, dem Einzelnen Orientierung und Halt zu geben.

Wenn nun diese frühere Identifikation mit dem System von anderen (Menschen oder Gruppen in Ost- und Westdeutschland) abgelehnt wird, und wenn man darüber hinaus "gezwungen" ist, diese Identifikation nunmehr selbst abzulehnen (obwohl man eigentlich mehr oder weniger stark von den alten Werten überzeugt ist), um in dem neuen System Anerkennung zu finden, so füht dies $z u$ erheblichen Identitäts- und Anpassungsproblemen. Hier ist allerdings zu unterscheiden zwischen einer Identifika- 
tion mit der politischen Ideologie und einer Identifikation mit dem Staat; bei letzterer sind weniger intensive selbstwertrelevante Probleme zu erwarten.

Weiter ist anzunehmen, daß umso weniger Identitätsprobleme aufgrund früherer Systemidentifikation bestehen, je enger (heute) die Einbindung in eine gleichdenkende soziale Gruppe ist, die die Funktion der früheren Bezugsgruppe übernimmt und psychologische Unterstützung leistet. So ist zu vermuten, daß PDS-Angehörige diese Identitätsprobleme weniger stark erleben, da sie einen wesentlichen Teil ihrer bisherigen Identität im Rahmen der Partei konservieren können.

Das Bedürfnis nach Identitätsfindung und -erhaltung hängt vom Entwicklungsalter ab und ist besonders bei Jugendlichen ausgeprägt (vgl. Erikson, 1988; Havighurst, 1973). Unter Belastungsbedingungen bevorzugen Jugendliche bizarre und extreme Lösungen ihrer Identitätsprobleme. So bewältigen ostdeutsche Rechtsradikale Identitätsprobleme, indem sie eine neue politische und soziale Identität in einer deutschen Nationalität suchen. Dabei wird der bisher bewährte Mechanismus eingesetzt, der die Abgrenzung gegen einen äußeren Feind und damit eine Selbstwerterhöhung sichert. Anstelle des Klassenfeindes erfolgt die Abgrenzung gegen Ausländer und Asylanten. Für beide Beispiele ist bezeichnend, daß die Identifikation mit einer hoch bewerteten sozialen Bezugsgruppe eine schonende Umstellung vom sozialistischen Staat der DDR auf das System der Bundesrepublik erlaubt.

Systembewertung und Identitat. Das System der ehemaligen DDR und das der Bundesrepublik kann vom Einzelnen also jeweils ganz unterschiedlich bewertet werden, eher in Übereinstimmung mit eigenen Werthaltungen, Wünschen, Zielen und bisher aufgebauten Fähigkeiten, oder in Diskrepanz zu solchen selbstwertrelevanten Merkmalen. Es sind jedoch auch ganz ambivalente Bewertungsergebnisse aufgrund widersprüchlicher Präferenzen wahrscheinlich. Bei geringer Übereinstimmung mit den Werthaltungen der Bundesrepublik, bei hoher Identifizierung mit dem System der ehemaligen DDR sowie bei hoher Investition in Fähigkeiten, die in der ehemaligen DDR honoriert wurden, die aber in der Bundesrepublik eher abgelehnt werden, sind besondere Anpassungsprobleme zu erwarten.

Die subjektive Wahrnehmung und Bewertung der jeweiligen Systeme ist also auch bedeutsam für individuelle Erwartungen und die eigene Identität. Daher bestehen hier psychologische Ausgangsbedingungen für den individuellen Umgang mit den Transformationsprozessen.

Wenn die Funktion der subjektiven Bewertung der Systeme für die Anpassung an den Wandel und für die Transformation (auch in den Beiträgen dieses Bandes) nicht direkt empirisch untersucht wurde, so mag dies auch an der Schwierigkeit liegen, zuverlässige Daten über die Systembewertung vor allem zum Zeitpunkt der Wende zu gewinnen. Heute lassen sich solche Daten nur durch retrospektive Verfahren erheben, was natürlich erhebliche methodische Probleme mit sich bringt. Subjektive Systembewertung und deren Identitätsrelevanz bedürfen als besondere psychologische Ausgangsbedingungen für die Transformationsprozesse eingehender Untersuchungen. Hier lassen sich subjektive Orientierungen und institutionelle Transformationen in ihrer wechselseitigen Beziehung erkennen. 


\subsection{Identitätsprobleme durch Selbstbewertung}

Systembewertungen hängen also mit Selbstbewertungen zusammen. Die Anpassungsanforderungen können besonders tiefgreifend sein, wenn negative System- oder auch Selbstbewertungen die eigene Identität belasten. Selbstbewertungen können u.a. auf internen und auf sozialen Vergleichen beruhen.

Interne Vergleiche. Bewertung eigner Erfahrungen: Bei internen Vergleichen stellen bisherige Werte und Erfahrungen den Bezugsanker dar. Solche Vergleiche können zu erheblichen Identitätsproblemen führen. Manche Menschen haben sich (trotz Vorbehalten) zu Zugeständnissen und Anpassungen im DDR-Regime bereit erklärt, um andernfalls unzulängliche Privilegien zu erhalten; sie haben damit eigene Werthaltungen in Frage gestellt. Umgekehrt haben andere damals Benachteiligungen riskiert, um eigene Überzeugungen zu vertreten. In beiden Fällen hat man aus heutiger Sicht unnötig viel aufs Spiel gesetzt. Selbstwertrelevante Fragen sind, was von den damaligen Überzeugungen und Handlungen heute noch als wichtig erlebt und entsprechend vertreten wird. Hat man sich früher für falsche Ziele engagiert? Waren die früher bzw. bisher vertretenen Überzeugungen tatsächlich wertlos? Muß man sich als Anpasser verachten?

Bewertungen bisheriger Investitionen: Über Fragen zu Werten und Überzeugungen, die heute nicht mehr akzeptabel sind, hinaus werden Fragen nach bisher als wertvoll und wichtig erachteten Leistungen und Investitionen, die heute nicht mehr gelten, gestellt. Hat man mit der bisherigen Ausbildung und Tätigkeit Kompetenzen aufgebaut, die sich jetzt als überflüssig und untauglich, ja sogar als hinderlich für ein erfolgreiches Bestehen nach der Wende herausstellen? Hat man mit diesen Fehlinvestitionen nicht nur für sich selbst, sondern auch für die eigene Familie unabsehbaren Schaden angerichtet?

Hier bestehen erhebliche selbstwertrelevante Diskrepanzen zwischen früher allgemein hochgeschätzten und dem im heutigen System bevorzugtem Verhalten. Die bisherige Sozialisation und die wichtigen Bezugsgruppen haben die Herausbildung solcher Werthaltungen und Verhaltensstile bedingt, die für die eigene Identität zentral waren, aber heute zu Anpassungsschwierigkeiten führen. Bei der Wahrnehmung fehlinvestierter selbstrelevanter Werthaltungen, Leistungen und Ressourcen sind identitätsbedrohende Verlusterlebnisse und Enttäuschungen zu verarbeiten. Auch Ziele, die bisher als bedeutsam und realisierbar verfolgt wurden, erscheinen nunmehr unerreichbar. In solche Bewertungen können negative und selbstwertbedrohliche Bilanzierungen von Kompensationsmöglichkeiten bisher investierter Ressourcen eingehen (vgl. Baltes \& Baltes, 1989; 1990). Diese selbstwertrelevanten Diskrepanzerfahrungen können die Grundlagen und Hierarchie des Selbstsystems (Epstein, 1980) in Frage stellen.

Soziale Vergleiche. Bei der Selbstbewertung werden über interne Vergleiche mit bisherigen Erfahrungen hinaus soziale Vergleiche durchgeführt. Eigene Erfahrungen und Bewertungen werden mit denen von anderen Menschen, insbesondere mit relevanten Personen und sozialen Gruppen verglichen bzw. an dem daraus resultierenden Anspruchsniveau gemessen. Je nachdem, mit wem man sich vergleicht, erlebt man 
selbstwertbelastende Ungerechtigkeit (vgl. Montada, in diesem Band). Warum sind andere (sowohl in der ehemaligen DDR wie aber auch in den alten Bundesländern) in der Zeit vor und jetzt nach der Wende erfolgreicher gewesen? Warum mußte man selbst die Belastungen in dem sozialistischen System ertragen? Warum wird man dafür jetzt nicht entschädigt anstatt unfreiwillig weitere neue Belastungen auf sich nehmen zu müssen, während Bewohner der alten Bundesländer schon lange in Wohlstand, Freiheit und Demokratie leben (und außerdem von der ungerechten Verteilung gegenwärtig profitieren)? Eine so aufgebaute "Opfermentalität" kann durch ein entsprechendes soziales Umfeld (Medien etc.) verstärkt und zur allgemeinen Überzeugung im Sinne kollektiver Selbststereotypisierung werden.

Ein negativer Selbstwert kann auch durch die wahrgenommene negative Bewertung der eigenen sozialen Gruppe (vor allem, wenn man sich mit ihr identifiziert) vermittelt werden und identitätsbelastend sein. Andererseits können negative Stereotypisierungen durch andere Gruppen die Einbindung in die eigene Gruppe fördern und entsprechenden Identitätsschutz zur Folge haben (vgl. Tajfel, 1982; Tajfel \& Turner, 1979). Dies mag eine der Funktionen der Selbst- und Fremdbezeichnung als "Ossi" und "Wessi" sein.

Handlungsrelevanz. Interne und soziale Vergleiche sind Grundlage selbstwertrelevanter Urteile, die eine wichtige Rolle im Umgang mit den Umbrüchen haben. Dabei hängen selbstwertrelevante Urteile auch von Ursachenzuschreibungen $\mathrm{ab}$. Je nachdem ob enttäuschte Hoffnungen oder Ungerechtigkeit als vorübergehend oder dauerhaft, als selbst- oder fremdverschuldet, als vermeidbar oder unausweichlich erscheinen, werden entsprechende selbstwertrelevante Emotionen (Ärger, Frustrationen), Motive sowie auch Verhalten ausgelöst. Wenn enttäuschte Erwartungen als willkürliche Schädigung eigener Interessen durch andere gedeutet werden und die generalisierte Überzeugung entsteht, Opfer von Ungerechtigkeit zu sein (z.B. weil eigenes Bemühen nicht die "verdienten" Erfolge bringt), sind anomische Reaktionen möglich (zur Aggression vgl. Kornadt, 1992). So belegen Golz et al. (1992) in einer von der Kommission für die Erforschung des sozialen und politischen Wandels in den neuen Bundesländern e.V. (KSPW) geförderten Studie, daß jugendliche Sympathisanten rechter Gruppen sich als Opfer von Ungerechtigkeit fühlen und daher Gewalt bejahen. Gewalt dient dem Ziel, sich gegen erfahrenes Unrecht zu wehren.

Die selbstwertbelastende Wahrnehmung und Beurteilung vergangener und gegenwärtiger Gegebenheiten kann zu Diskrepanzerlebnissen und Frustrationen in zentralen subjektiv bedeutsamen Wertbereichen sowie zu Identitätskrisen führen. Wie diese schließlich verarbeitet werden (z.B. Gewalt, soziale Isolation), hängt u.a. davon ab, wie zentral und selbstwertrelevant die persönlichen Ziele und Werte sind, die von den erforderlichen Umbewertungen betroffen sind, wie ausgeprägt die Unvereinbarkeit der bisherigen Orientierungen und der neuen Anforderungen erlebt wird, und in welchem sozialen Kontext man eingebettet ist.

Aus theoretischen und praktischen Gründen sollten die Bedingungen, Formen und Verarbeitungsprozesse von Selbstwert- und Identitätsproblemen genauer studiert werden, auch weil in der gegenwärtigen Transformationsforschung das Identitätskonzept als Sammelbegriff für individuelle Orientierungen inzwischen inflationär behandelt wird. Die Verarbeitung von Selbstwert- und Identitätsproblemen verdient in zukünftiger Transformationsforschung besondere Aufmerksamkeit. Hier erfolgen Prozesse, 
die in besonderer Weise die Verbindung zwischen Individuum und Gesellschaft im Transformationsprozeß zu analysieren erlauben. Die veränderten gesellschaftlichen Bedingungen wirken sich auf die Identität der Menschen aus, während die Identität dieser Menschen ihrerseits die gesellschaftlichen Bedingungen beeinflußt (Rosenberg, 1979). Identität ist danach ein Ergebnis sozialer Bedingungen und wirkt gleichzeitig auf soziale Prozesse ein (Identität als "social product" und "social force"). So wirken z.B. Personen, die sich aufgrund von Identitätsproblemen bestimmten sozialen Grup pen mit identitätsstiftenden und -stabilisierenden Funktionen anschließen, aktiv auf weitere Transformationsprozesse ein.

\subsection{Individuelle Verarbeitung von Herausforderungen}

\subsubsection{Kontroll- und Zukunftsorientierung als Verarbeitungsformen}

Wie der Einzelne die Umbrüche und die davon ausgelösten Ambivalenzen und Unsicherheiten nach dem Beitritt verarbeitet, hängt u.a. mit den Faktoren zusammen, die z.T. im bisherigen Abschnitt besprochen wurden, d.h. seiner bisherigen Sozialisation und der in deren Verlauf aufgebauten Identität mit den damit verbundenen Handlungsorientierungen, Zielen und Kompetenzen sowie der gegebenen Einbindung in bestimmte soziale Netze und Bezugsgruppen. Einerseits werden diese Faktoren durch den Wandel mit beeinflußt; andererseits sind dies auch die Ausgangsbedingungen für die Verarbeitung von transformationsbedingten Herausforderungen (Belastungen und Chancen). Im folgenden soll nur ein Bereich dieser Anpassungsbedingungen - die Kontrollorientierung als Teil der allgemeinen individuellen Handlungsorientierung - skizziert werden. Hier wird angenommen, daß Kontrollorientierung einerseits kognitiv-motivationale Anpassungsformen vermittelt und andererseits durch die erlebten Diskrepanzen auf die Probe gestellt und ggfs. modifiziert wird. Somit werden durch die Kontrollorientierung die wechselseitigen Einflüsse zwischen Individuum und sozialem Wandel vermittelt.

Nach Kagan (1981) besteht ein Grundbedürfnis des Menschen darin, Sicherheit zu gewinnen. Dies liegt vermutlich dem universellen menschlichen Ziel zugrunde, Kontrolle zu erleben. Bei Erfüllung dieses Zieles kann eine positive Kontrollorientierung und optimistische Zukunftserwartung entstehen. Die Funktion von Kontrollorientierung besteht im wesentlichen in der Überzeugung, daß Ereignisse gemäß bestimmten Bedingungen mit gewisser Regelhaftigkeit auftreten, also mehr oder weniger direkt vorhersagbar und ggfs. auch steuerbar sind.

Aus der Motivations- und Entscheidungsforschung (z.B. Heckhausen, 1989) ist bekannt, daß Kontrollüberzeugungen (z.B. die Art der Ursachenzuschreibung für erlebte Ereignisfolgen wie eigene Erfolge und Mißerfolge) und Zukunftserwartungen das menschliche Handeln grundlegend beeinflussen. Diese kognitiv-motivationalen Prozesse erleichtern eine konstruktive Gestaltung der eigenen Lebensumwelt auch unter erschwerten Bedingungen. Kontrollüberzeugung und Zukunftszuversicht erlauben, Frustrationen und Schwierigkeiten erfolgreich zu überwinden. So kann eine hohe Kontrollüberzeugung (Probleme werden als lösbar wahrgenommen) und eine optimistische Zukunftserwartung die Bereitschaft zu Leistungshandeln, vorübergehendem Belohnungsverzicht, zu Investition und Ausdauer bei Problembewältigung fördern 
(vgl. Scheier \& Carver, 1985). Eine Reihe von Studien zeigen (z.B. Bandura, 1986), $\mathrm{daB}$ die Überzeugung von eigener Kontrolle und Selbstwirksamkeit mit hoher Problemlösefähigkeit, sozialen Kompetenzen und "coping"-Strategien zusammenhängt.

Erfahrungen von erlebter Kontrolle, wie dies in der Zeit der Wende und des Beitritts für Ostdeutsche der Fall gewesen sein dürfte, müßten positive Kontrollüberzeugungen und Selbstwirksamkeitsurteile beeinflussen. Dies wiederum müBte die Bereitschaft stärken, Probleme als Herausforderung zu erleben und nach Lösungen zu suchen. Umfrageergebnisse von Habich, Landua und Priller (1991), die zeigen, daß trotz geringer Lebenszufriedenheit ein hoher Optimismus bei Ostdeutschen bestand, entsprechen diesen theoretischen Überlegungen.

Wie weit allerdings durch die dann folgenden Umbrüche und möglichen Diskrepanzerlebnisse diese positive Kontroll- und Zukunftsorientierung und die dadurch beeinflußte Frustrationsfähigkeit belastet werden kann, ist unklar. Die Kontrollorientierung und die dadurch vermittelten Anpassungsprozesse müssen bei dem ständigen Wandel von Anforderungen selbst nicht stabil bleiben. Wenn wiederholt Ungerechtigkeit und Kontrolleinschränkung erfahren wird, kann bei entsprechenden Attribuierungen (Selbstbewertung als ungerecht behandeltes "Opfer"; als "Spielball" anderer) ein Kontrollverlust erlebt werden. So verliert man schließlich das Vertrauen in eigene Fähigkeiten und in die gerechte Welt. Dies hat demotivierende Effekte und kann zur Resignation und Apathie führen. Zumindest wird die Bereitschaft zur Investition eigener Ressourcen (z.B. bei Weiterbildungsentscheidungen) oder zu Verzichtleistungen reduziert.

$\mathrm{Da}$ Kontroll- und Zukunftsorientierung wesentliche Voraussetzungen für Entscheidungen und problemlösendes Handeln bzw. für Verarbeitungsprozesse sind, ist $\mathrm{zu}$ fragen, welche Änderungen sich durch die Transformationsprozesse in diesen Orientierungen ergeben, wie sich dies auf individuelles Handeln auswirkt und ob es zu Bewältigungsproblemen kommt. Dazu sind empirische Verlaufsstudien erforderlich.

\subsection{2 "Primäre" und "sekundäre" Kontrollorientierung im Transformationsprozeß}

Zum Konzept. Es liegen inzwischen eine Vielzahl von theoretischen Ansätzen zur Kontrollüberzeugung und ihrer Handlungsrelevanz vor (vgl. von Cranach, 1991). Hier soll ein neuerer Ansatz hervorgehoben werden, der sich für die Analyse von subjektiver Wahmehmung und Verarbeitung von Umbruchsituationen besonders gut zu eignen scheint, der von Rothbaum, Weisz und Snyder (1982) und Weisz, Rothbaum und Blackburn (1984) entwickelte Ansatz der "primären" und "sekundären" Kontrollorientierung. Die Autoren nehmen an, daß Kontrollorientierung eine Überzeugung ist, nach der eher die Umwelt gemäß eigenen Zielen geändert wird ("primäre" Kontrolle), oder nach der eigene Ziele gemäß Gegebenheiten der Umwelt geändert werden ("sekundäre" Kontrolle). Entsprechend bestehen unterschiedliche Überzeugungen, wie eine Passung von Erwartungen und Umwelt möglich ist (Trommsdorff, 1989b; 1993b).

Beide Kontrollarten verhindern jeweils auf ihre Art das lähmende Bewußtsein von Kontrollverlust und können die Überzeugung von Kontrolle stärken. Für Menschen, 
die in Umbruchsituationen hohe Belastungen zu verarbeiten haben, können beide Kontrollorientierungen (situationsspezifisch) eine konstruktive Möglichkeit zur Bewältigung von Schwierigkeiten bedeuten. Jedoch bestehen individuelle Unterschiede darin, ob eher die eine oder andere Kontrollform bevorzugt wird. Außerdem haben sich deutliche Kulturunterschiede in der Bevorzugung von "primärer" und "sekundärer" Kontrolle gezeigt: in sozialorientierten (asiatischen) Kulturen besteht eher "sekundäre" und in individualorientierten (westlichen) Kulturen eher "primäre" Kontrolle (vgl. Trommsdorff, 1989b; Seginer, Trommsdorff \& Essau, 1993; Essau \& Trommsdorff, 1993). Dies spricht für die Wirksamkeit kultureller Werte und Sozialisationseinflüsse. Schließlich ist offensichtlich, daß die Bevorzugung der einen oder anderen Kontrollorientierungen von dem jeweiligen situativen Kontext und Bereich abhängt (Trommsdorff, 1993b).

In einer Phase massiven Umbruchs des gewohnten Lebenskontextes und der Infragestellung von bisherigen subjektiven Zielen und Werten werden vermutlich auch bisherige Prioritäten von "primärer" und "sekundärer" Kontrollorientierung in Frage gestellt, in bezug auf ihre adaptive Funktion überprüft und möglicherweise in ihrer Gewichtung verändert (vgl. Trommsdorff, 1993b). Ob nun bei der subjektiven Bewältigung von transformationsbedingten Belastungssituationen im individuellen Handlungsverlauf "sekundäre" auf "primäre" Kontrollstrategien folgen, ob beide gleichzeitig (mit gleicher oder verschiedener Gewichtung) eingesetzt werden, oder ob beide je nach kulturellen Werthaltungen und Sozialisationsbedingungen - kulturspezifisch bei unterschiedlicher Priorität unterschiedlich ausgeprägt sind: dies ist empirisch zu prüfen.

Ausgangsbedingung für "primäre" und "sekundäre" Kontrollorientierung. Welche Ausgangsbedingungen in bezug auf Kontrollorientienung in der ehemaligen DDR bestanden haben, läßt sich schwer abschätzen. Der aufschlußreiche Vergleich zweier Theorien zum DDR-System von K.U. Mayer (1993) läßt beide Varianten zu - die Bevorzugung von "sekundärer" Kontrolle wegen hohen Anpassungsdrucks auf das "Herrschaftssubjekt" (De-Subjektivierungsthese), aber auch die Bevorzugung von "primärer" Kontrolle wegen der aktiven Nutzung informeller Strukturen mit vielfältigen Handlungsstrategien (Neo-Traditionalismus-These).

In der ehemaligen DDR konnte "primäre" Kontrolle durch Vergabe von Privilegien erfolgen. Menschen, die sich in dem System andere gefügig gemacht haben, indem sie sie in den Dienst eigener Ziele gestellt haben, würden danach "primäre" Kontrolle ausgeübt haben. Sie haben sich z.B. in Funktionärpositionen gebracht, die ihnen Macht über andere Personen gegeben haben. Damit haben sie Mittel der "primären" Kontrolle verwendet. Andere haben sich dem System gefügt und ihre Interessen, Ziele, Wünsche und Fähigkeiten dem System untergeordnet; sie haben sich im Sinne "sekundärer" Kontrolle den Gegebenheiten angepaßt. Der hohe Wert von Solidarität und Hilfsbereitschaft, der sich in zahlreichen Studien nachweisen läßt (z.B. Sturzbecher, 1993), könnte auch ein Indikator für "sekundäre" Kontrollorientierung sein.

Durch die Wende ist die "primäre" Kontrollüberzeugung aufgewertet worden. Bereits die Deutung einer Selbstverursachung der Wende legt eine "primäre" Kontrollüberzeugung nahe. Ostdeutsche sehen sich z.B. auf dem Arbeitsmarkt und im Betrieb vor die Aufgabe gestellt, "Initiative" zu ergreifen und "Selbstdarstellung" zu zeigen (vgl. Rappensperger, Rosenstiel \& Zwarg; Frese \& Hilligloh; Wottawa, in diesem 
Band). Diese Verhaltensweisen entsprechen den Merkmalen der "primären" Kontrollüberzeugung, werden von Ostdeutschen nach diesen Studien aber eher abgelehnt.

Einer verstärkten Präferenz von "primärer" Kontrolle stehen gegenwärtig wohl nicht nur die Sozialisationserfahrungen, sondern auch die vielfaltigen Unsicherheiten und Einschränkungen von "primärer" Kontrolle (insbesondere auf dem Arbeitsmarkt) entgegen, die seit dem Beitritt für Ostdeutsche entstanden sind. Eine aus Westdeutschland importierte Abwertung von "sekundärer" Kontrolle bei gleichzeitigen Schwierigkeiten, "primäre" Kontrolle zu übernehmen, hätte problematische Folgen bis hin zum Erleben von Kontrollverlust. Die Erwartung an Ostdeutsche, einerseits die Umbrüche (mit den Belastungen) zu akzeptieren und andererseits Aktivität und Eigeninitiative zu zeigen, dürfte auch nicht unproblematisch sein, da dies eine ambivalente Kontrollorientierung zur Folge haben kann. Diese Ambivalenz könnte allerdings durch eine situationsspezifische flexible Differenzierung (in bestimmten Situationen wird eher "primäre", in anderen eher "sekundäre" Kontrollüberzeugung bevorzugt) aufgefangen werden. $\mathrm{Ob}$ dies gelingt, müßte in Längsschnittstudien geprüft werden. Damit ließen sich aufschlußreiche Einblicke in die Wechselwirkungsprozesse gesellschaftlich bedingter Änderungen von individueller Kontrollorientierung und deren Wirkung auf individuelles Handeln und Bedingungen sozialen Wandels gewinnen.

\section{Ergänzende theoretische und methodische Aspekte}

In das Studium psychologischer Aspekte von Transformationsprozessen gehen (kulturgebundene) subjektive Theorien implizit in Fragestellung, Erhebung und Dateninterpretation ein. Ein westdeutscher Forscher, der glaubt, "unbetroffen" bzw. neutral zu sein, muß seine Voreingenommenheiten genauso präzise artikulieren wie der ostdeutsche "betroffene" Forscher, der aufgrund seiner intimen Kenntnis der Gegebenheiten der ehemaligen DDR glaubt, eine "realitätsnähere" Beschreibung und Dateninterpretation liefern zu können. Wie in anderer sozialwissenschaftlicher Forschung sind auch hier Bezugsgruppeneffekte und Voreingenommenheiten wirksam, die die Generierung und Interpretation der Daten beeinflussen.

Vergleiche zwischen ost- und westdeutscher Bevölkerung erscheinen manchmal von Voreingenommenheiten und Wunschvorstellungen der Autoren geprägt, sei es, daß Gemeinsamkeiten als Beleg für die gemeinsame deutsche Kultur und Geschichte und Unterschiede als Beleg für die verschiedene Vergangenheit in unterschiedlichen Systemen und für Schwierigkeiten bei der Anpassung genommen werden.

Eine schlichte Beschreibung der Transformationsprozesse und der subjektiven Orientierungen in Ostdeutschland allein sagt für sich genommen nicht viel aus, wenn nicht vergleichend vorgegangen wird. Viele Belastungen (und deren Akkumulationen) können in Ost- und Westdeutschland zunächst gleich sein (z.B. Arbeitslosigkeit von ungelernten Arbeitern); eine psychologische Transformationsforschung muß jedoch die spezifischen Besonderheiten in Ost- und Westdeutschland vergleichend herausarbeiten. Dazu sind zunächst die psychologischen Ausgangsbedingungen für den Umgang mit den Transformationsprozessen systematisch zu beschreiben, um dann zu untersuchen, wie die Anforderungen unter den jeweiligen Bedingungen in Ost- und Westdeutschland individuell verarbeitet werden. 
Im übrigen sind bei allen Vergleichen bestimmte methodische Voraussetzungen zu erfüllen. Das gilt für Vergleiche innerhalb der ostdeutschen Bevölkerung, um Veränderungen festzustellen; dies gilt auch für Vergleiche zwischen der ost- und westdeutschen Bevölkerung, um durch Unterschiede und Gemeinsamkeiten die jeweiligen Ausgangsbedingungen und die Anpassungen an die Transformationsprozesse und den Wandel zu erfassen, und z.B. um Annäherungen und Distanzierungen zwischen der Bevölkerung der beiden Teile Deutschlands im Zeitverlauf zu prüfen.

Die kühne Hoffnung, mit der Wende nunmehr ein "quasi-natürliches Experiment" zur Untersuchung von individuellen Anpassungsprozessen an Transformationsprozesse sowie gleichzeitig Kulturvergleiche durchführen zu können, muß angesichts der Komplexität der den Transformationsprozeß begleitenden Umstände und der damit verbundenen theoretischen und methodischen Anforderungen etwas nüchterner gesehen werden. Der Vergleich von relativ stabilen kognitiven Orientierungen in Ostdeutschland wie individuellen Zielen und Werthaltungen kurz vor und kurz nach der Wende ist etwas kurzsichtig. Zunächst ist die "Wende" ein theoretisch diffuses und hinsichtlich ihrer Einflufgrößen nicht spezifiziertes Phänomen. Sicher kann der Mauerfall oder der Beitritt allein nicht als die eigentlich theoretisch relevante Einflußgröße (für ein "Vorher-Nachher-Exeriment") gelten, u.a. weil bereits vor der Wende Wandlungsprozesse erfolgt sind, oder weil die Währungsunion als der eigentlich relevante Umbruch gesehen werden kann.

Auch der Anspruch, Vergleiche zwischen ost- und westdeutschen Stichproben gemäß Methoden des klassischen Kulturvergleichs (vgl. Berry et al., 1992) durchführen zu können, erscheint wegen der ungenauen Kenntnis der Ausgangsbedingungen in Ostdeutschland und wegen der intrakulturellen Varianz in den neuen und alten Ländern schwer erfüllbar.

Intrasystemische Varianz. Der Systemumbruch hat sich keineswegs für alle Bevölkerungsgruppen in den neuen Bundesländern in gleicher Weise ausgewirkt, u.a. wegen beträchtlicher regionaler Unterschiede der Wirtschaftsstruktur, des Arbeitsmarktes etc. (vgl. Bertram, Bayer \& BauereiB, 1993). Außerdem sind, wie bereits skizziert, die psychologischen Aspekte der Transformationsprozesse im Zusammenhang mit dem sozialen Kontext, der subjektiven und objektiven Lebenslage, der Kohortenzugehörigkeit, dem Entwicklungsalter sowie Geschlecht und Bildung zu differenzieren. Die dadurch jeweils vermittelten Sozialisations- und Handlungs-bedingungen können die Wirkungen der Transformationsprozesse (z.B. die Chancen und Risiken) verstärken oder nivellieren.

Damit stellen sich Probleme der Stichprobenwahl und Fragen nach der Generalisierbarkeit der Ergebnisse. Auch wenn für viele psychologische Fragestellungen nicht erforderlich ist, bevölkerungsrepräsentative Stichproben zu untersuchen, ist zu präzisieren, was diese Stichproben theoretisch repräsentieren, und wie weit die Befunde generalisierbar bzw. auf welchen Bereich sie einzuschränken sind. Während Umfragen meist auf Aggregatniveau analysiert und interpretiert werden, sind bei Analysen zu psychologischen Prozessen vor allem die jeweiligen individuellen Zusammenhänge sowie auch die Durchschnittswerte in Verbindung mit der individuellen Abweichung vom Mittelwert (Streuung) von Interesse. Daher kann es sinnvoll sein, andere Gruppierungen vorzunehmen, als dies bei soziologischen Fragestellungen sinnvoll ist. Nach den obigen Überlegungen können z.B. Gruppierungen von Personen nach Vari- 
ablen erforderlich sein, die für den Umgang mit transformationsbedingten Belastungen zentral sind - also z.B. Vergleiche zwischen Personen mit unterschiedlicher Kontrollund Zukunftsorientierung.

Validitat der Befragungsergebnisse. Daß die Antwortbereitschaft in den neuen im Vergleich zu den alten Bundesländern erheblich höher ist, haben die meisten bisherigen Vergleichsstudien gezeigt. Ob dies nun ein höheres AusmaB an "ehrlichen", z.B. von sozialen Erwünschtheitstendenzen nicht beeinträchtigten Antworten bedeutet, ist ungeklärt. Hier stellt sich also die Frage, ob in beiden Teilen Deutschlands mit den gleichen Verfahren auch tatsächlich das gleiche Konstrukt gemessen wurde. Die Sicherstellung der Äquivalenz von Meßverfahren soll vor allem die Validität der Verfahren gewährleisten (vgl. Trommsdorff, 1989a). Zu prüfen ist darüber hinaus, ob die Antworten in beiden Bevölkerungsgruppen auch gleichermaßen valide sind, bzw. ob unter den gleichen Fragen in beiden Teilen Deutschlands das Gleiche verstanden wird, also ob die Verfahren funktional äquivalent sind.

Funktionale Äquivalenz. In diesem Zusammenhang besteht das Problem, daß formal gleiche Antworten, wie hohe individualistische Wertorientierung (z.B. eigene Unabhängigkeit) bei Ost- und Westdeutschen nicht das Gleiche bedeuten müssen. Einzelne Einstellungen sind Facetten eines Systems; sie sind keineswegs als isolierte Phänomene wirksam, sondern sie sind in ein individuelles System von Werthaltungen und Handlungstendenzen sowie in einen bestimmten sozialen Kontext eingebettet. Entsprechend haben einzelne Werte für verschiedene Individuen je nach deren Wertsystem (bzw. dem Status einzelner Werte darin) spezifische, unterschiedliche Bedeutungen, auch wenn der einzelne Wert formal gleich erscheint. Je nach individueller Erfahrung und Einbettung in das soziale Umfeld, das wiederum bestimmte Werthaltungen vertritt, kann das individuelle Wertsystem und ein spezifischer Wert darin eine andere Bedeutung, Stabilität oder Ambivalenz haben (Trommsdorff, 1993a). In der ehemaligen DDR waren z.B. materielle Werte offiziell von geringer, aber privat für den einzelnen von hoher Wichtigkeit. Materielle Werte scheinen nach Umfragen für (altersund bildungsgleiche) Westdeutsche weniger relevant als für Ostdeutsche zu sein. Sind die Wertsysteme wirklich verschieden oder sind nur einzelne Werte darin gegenwärtig unterschiedlich bedeutsam, z.B. weil sie bisher in unterschiedliche Systeme eingebunden waren? Ähnlich vorsichtig sollten daher auch Konzepte verwendet werden, die zur Beschreibung von Lebensstilmerkmalen (wie z.B. pluralistische Lebensform) im Westen brauchbar sein können, deren Anwendbarkeit für den Osten Deutschlands jedoch zu klären ist. Daher ist zu prüfen, was die auf Aggregatebene nachgewiesenen Ähnlichkeiten und Unterschiede von singulären Merkmalen bedeuten, welche Merkmalskombinationen beim Einzelnen bestehen und vor allem welche Verhaltensrelevanz diese haben.

Um teilweise Widersprüche in bezug auf Unterschiede und Ähnlichkeiten in der ost- und westdeutschen Bevölkerung bei den verschiedenen Surveys aufzuklären, reicht es nicht, Teilstichproben (nach Alter, Geschlecht etc. differenziert) zu vergleichen. Vielmehr ist die eigentlich interessante Frage, welche Bedeutung solche Gemeinsamkeiten und Unterschiede als psychologische Merkmale für den Einzelnen im Transformationsprozeß haben, d.h. wie der soziale und wirtschaftliche Umbruch aufgrund dieser Merkmale vom Einzelnen erlebt und verarbeitet wird. Ob die Verarbei- 
tungsprozesse von Ostdeutschen eher durch ähnliche Werthaltungen und Zukunftserwartungen, wie sie in Westdeutschland bestehen, erleichtert werden oder nicht, wäre dabei zu untersuchen. Ähnlichkeit und Unähnlichkeit von Merkmalen in Ost- und Westdeutschland festzustellen, besagt also wenig, wenn die Funktion dieser Merkmale fü die Bewaltigungsstrategien im Transformationsproze $B$ unklar ist. Um diese Funktion zu untersuchen, sind eigene Studien erforderlich, die möglichst als Längsschnitt angelegt, und über den deutsch-deutschen Vergleich hinaus weitere Personengruppen einbeziehen sollten, die in ganz anderen kulturellen und sozioökonomischen Kontexten (z.B. in Osteuropa) ähnliche Transformationsprozesse erleben.

Forschungsstrategisch ist das Paradigma des Kulturvergleichs auch aufgrund der kulturellen Gemeinsamkeiten in Ost- und Westdeutschland sinnvoll, um gesellschaftlich relevante psychologische Bedingungen im Verlauf des massiven sozialen, wirtschaftlichen und politischen Wandels zu untersuchen. Allerdings müssen dazu die Bedingungen, in denen die Menschen aus beiden Teilen Deutschlands aufgewachsen sind und gegenwärtig leben, in den Vergleich einbezogen werden. Ohne präzise Beschreibung dieser Bedingungen lassen sich keine angemessenen Vergleiche durchführen und keine funktional äquivalenten Verfahren entwickeln. Dies gilt auch, wenn sich im Transformationsprozeß laufend die gegenwärtigen Bedingungen ändern. Umso wichtiger ist es, wenigstens mit "Momentaufnahmen" der Transformationsprozesse zu beginnen. Gegenwärtig erscheinen diese in Ausmaß und Richtung schwer einschätzbar, vor allem, wenn diese in einem größeren Kontext des sich änderen Europa gesehen werden.

In diesem Beitrag sollte wenigstens darauf hingewiesen werden, daß die Transformationsprozesse durch individuelle Handlungsorientierungen und damit verbundenes Handeln mit beeinflußt werden, wie sie umgekehrt auch ihrerseits auf individuelles Handeln einwirken. Diese Wechselwirkungsprozesse zwischen gesellschaftlichen und individuellen Bedingungen im Transformationsprozeß bedürfen genauerer Analysen,

Neben diesen soll zum Schluß noch auf einen anderen Wechselwirkungsprozeß wenigstens hingewiesen werden, der von der Forschung bisher fast völlig vernachlässigt wurde: die Interaktionen zwischen den Menschen aus Ost- und Westdeutschland. Denn von diesen Menschen, ihren gegenseitigen Wahrnehmungen, ihrem Denken und ihrem sich darauf gründenden wechselseitigen Verhalten und ihrer Bereitschaft, psychologische Grenzen, Barrieren und Vorurteile zu überwinden, werden auch Richtung und Erfolg der Transformationsprozesse wesentlich mit abhängen.

\section{Literatur}

Bandura, A. (1986). The social foundations of thought and action: A social cognitive theory. Englewood Cliffs, NJ: Prentice Hall.

Baltes, P.B. \& Baltes, M.M. (1989). Optimierung durch Selektion und Kompensation: Ein psychologisches Modell erfolgreichen Alterns. Zeitschrift rür Pädagogik, 35, 85-105.

Baltes, P.B., \& Baltes, M.M (1990). Psychological perspectives on successful aging: The model of selective optimization with compensation. In P.B. Baltes \& M.M. Baltes (Eds.), Successful aging. Perspectives from the behavioral sciences (pp. 1-34). Cambridge: Cambridge University Press. 
Behnken, 1., Günther, C., Kabat vel Job, O., Keiser, S., Karig, U., Krüger, H.-H., Lindner, B., Wensierski, H.J. v. \& Zinnecker, J. (1991). Schülerstudie '90. Jugendliche im Prozel der Vereinigung. Weinheim: Juventa.

Bendix, R. (1965/66). Tradition and modemity reconsidered. Comparative Studies in Society and History, 9, 292-346.

Berry, J.W., Poortinga, Y.H., Segall, M.H., \& Dasen, P.R. (1992). Cross-cultural psychology; Research and applications. New York: Cambridge University Press.

Bertram, H., Bayer, H. \& Bauereiß, R, (1993). Familien-Atlas: Lebenslagen und Regionen in Deutschland: Karten und Zahlen. Opladen: Leske \& Budrich.

Bierhoff, H.W., Cohen, R.L., \& Greenberg, J. (Eds.) (1986), Justice in social relations. New York: Plenum.

Cranach, M. von (1991). Handlungsfreiheit und Determination als ProzeB und Erlebnis. Zeitschrift für Sozialpsychologie, 22, 4-24.

Dennhardt, R., Dippelhofer-Stiem, B., Eilders, C., Gille, M., Hoffmann-Lange, U., Krüger, W., Lange, G., Schubarth, W. \& Six, U. (1990). Deutsche Schüler im Sommer 1990 - Skeptische Demokraten auf dem Weg in ein vereintes Deutschland. Deutsch-deutsche Schülerbefragung 1990. München: DJI-Arbeitspapier 3-019.

Deutsches Jugendinstitut (Hrsg.) (1992). Schuller an der Schwelle zur deutschen Einheit. Politische und persönliche Orientierungen in Ost und West. Opladen: Leske \& Budrich.

Elder, G.H. (1979). Historical change in life pattem and personality. In P.B. Baltes \& O.G. Brim (Eds.), Life span development and behavior, Vol.2. (pp. 118-159). New York: Academic Press.

Epstein, S. (1980). The self-concept: A review and the proposal of an integrated theory of personality. In E. Staub (Ed.), Personality: Basic aspects and current research (pp. 82-131). Englewood Cliffs, N.J.: Prentice-Hall.

Essau, C. \& Trommsdorff, G. (1993, in Druck). Kontrollorientierung von Jugendlichen im Kulturvergleich. Zeitschrift für Sozialisation und Erziehungssoziologie. Schwerpunktheft Jugend im Kulturvergleich.

Erikson, E.H. (1988). Identität und Lebenszyklus. Frankfurt a.M.: Suhrkamp.

Festinger, L. (1957). A theory of cognitive dissonance. Standford, Calif.: Standford University Press.

Flanagan, C.A., \& Eccles, J.S. (1993). Changes in parents' work status and adolescents' adjustment at school. Child Development, 64, 246-257.

Friedrich, W. \& Griese, H. (Hrsg.) (1991). Jugend und Jugendforschung in der DDR. Gesellschaftspolitische Situationen, Sozialisation und Mentalitätsentwicklung in den achtziger Jahren. Opladen: Leske \& Buderich.

Gensicke, T. (1992). Mentalitätsentwicklung im Osten Deutschlands seit den 70er Jahren. Vorstellungen und Erläuterungen von Ergebnissen einiger empirischer Untersuchungen in der DDR und in den neuen Bundesländern von 1977 bis 1991. Speyerer Forschungsberichte Nr.109. Speyer: Forschungsinstitut für Öffentliche Verwaltung.

Gille, M. (1992). Lebensziele und Politikvorstellungen von Jugendlichen in Ost und West. In Deutsches Jugendinstitut (Hrsg.), Schüler an der Schwelle zur deutschen Einheit. Politische und persönliche Orientierungen in Ost und West (\$,18-41). Opladen: Leske $\&$ Budrich.

Golz, L. u.a. (Autorenkollektiv) (1992). Verbaltensdispositionen Jugendlicher in Neubrandenburg (Greifswald). Kurzstudie. Unveröffentlichtes Manuskript. Halle: Kommission fứr die Erforschung des sozialen und politischen Wandels in den neuen Bundesländern e.V. (KSPW).

Habich, R., Landua, D. \& Priller, E. (1991). Geringere Lebenszufriedenheit in der ehemaligen DDR. Erste Ergebnisse der empirischen Wohlfahrtsforschung. ISI-Informationsdienst Soziale Indikatoren, $5,1-4$.

Havighurst, R.J, (1973). A cross-cultural view of adolescence. In J.F. Adams (Ed.), Understanding adolescence: Current developments in adolescent psychology (2nd ed.). Boston, MA: Allyn \& Bacon.

Heckhausen, H. (1989). Motivation und soziales Handeln (2. Auflage). Berlin: Springer-Verlag.

Huinink, J. \& Mayer, K.U. (1993). Lebensläufe im Wandel der DDR-Gesellschaft. In H. Joas \& M. Kohli (Hrsg.), Der Zusammenbruch der DDR (S. 151-171). Frankfurt a.M.: Suhrkamp. 
Hurrelmann, K. (1986). Einführung in die Sozialisationstheorie. Über den Zusammenhang von Sozialstruktur und Persönlichkeit. Weinheim, Basel: Beltz.

Kagan, J. (1981). Universals in human development. In R.H. Munroe, R.L. Munroe, \& B. Whiting (Eds.), Handbook of cross-cultural human development (pp. 53-62). New York: Garland.

Komadt, H.-J. (1992). Trends und Lage der gegenwärtigen Aggressionsforschung. In H.-J. Komadt (Hrsg.), Aggression und Frustration als psychologisches Problem, Band 2 (S. 513-580). Darmstadt: Wissenschaftliche Buchgesellschaft.

Lazarus, R.S., \& Folkman, S. (1984). Coping and adaptation. In W.D. Gentry (Ed.), Handbook of behavioral medicine (pp. 282-325). New York: Guilford Press.

Lewin, K. (1935). Dynamic theory of personality. New York: MacGraw-Hill.

Mayer, K.U. (1993). Die soziale Ordnung der DDR und einige Folgen für die Inkorporation in die BRD. BISS Public, 11, 39-55.

Melzer, W., Lubowski, W. \& Schmidt, L. (1991). Deutsch-polnischer Jugendreport. Lebenswelten im Kulturvergleich. Weinheim: Juventa.

Reichardt, Ch. \& Schrōter, E. (1993). Berliner Verwaltungseliten. Rollenverhalten und Einstellungen von Führungskräften in der (Ost- und West-) Berliner Verwaltung. In W. Seibel, A. Benz, \& H. Mäding, (Hrsg.), Verwaltungsreform und Verwaltungspolitik im Prozeß der deutschen Einigung (S. 207-217). Baden-Baden: Nomos.

Reißig, R. (1993). Das Scheitern der DDR und des realsozialistischen Systems - Einige Ursachen und Folgen, In H. Joas \& M. Kohli (Hrsg.), Der Zusammenbruch der DDR (S.49-69). Frankfurt: Suhrkamp.

Rosenberg, M. (1979). Conceiving the self. New York: Basic Books.

Rothbaum, F., Weisz, J.R., \& Snyder, S.S. (1982). Changing the world and changing the self: A twoprocess modell of perceived control. Journal of Personality and Social Psychology, 42, 5-37.

Scheier, M.F., \& Carver, C.S. (1985). Optimism, coping, and health: Assessment and implications of generalized outcome expectancies, Health Psychology, 4, 219-247.

Schmidt, H.-D. (1992). Frühe Kindheit in der ehemaligen DDR im Spannungsfeld Familie/Krippe. Psychologie in Erziehung und Unterricht, 39, 149-155.

Seginer, R., Trommsdorff, G., \& Essau, C. (1993). Adolescent control beliefs: Cross-cultural variations of primary and secondary orientations. International Joumal of Behavioral Development, $16,243-260$.

Sturzbecher, D. (1993). Jugendliche in Brandenburg - Auf der Suche nach Orientienung. Vortrag aur Fachkonferenz "Entstehung von Fremdenfeindlichkeit - Die Verantwortung von Politik und Medien" der Friedrich-Ebert-Stiftung (22.03.1993). Potsdam.

Tajfel, H. (1982). Social identity and intergroup relations. London: Cambridge University Press.

Tajfel, H., \& Turner, C. (1979). An integrative theory of intergroup conflict. In G. Austin \& S. Worchel (Eds.), The social psychology of intergroup relations. Monterey, Calif: Brooks \& Cole.

Trommsdorff, G. (1989a). Kulturvergleichende Sozialisationsforschung. In G. Trommsdorff (Hrsg.), Sozialisation im Kulturvergleich (S,6-24). Stuttgart: Enke.

Trommsdorff, G. (1989b), Sozialisation und Werthaltungen im Kulturvergleich. In G. Trommsdorff (Hrsg.), Sozialisation im Kulturvergleich (S. 97-121). Stuttgart: Enke.

Trommsdorff, G. (1992). Forschung zur geschlechtsspezifischen Sozialisation in der damaligen DDR. Berliner Journal der Soziologie, 3, 389-398.

Trommsdorff, G. (1993a). Value change in Germany. A comparative perspective. In Japanstudien. Jahrbuch des Deutschen Instituts für Japanstudien der Philipp-Franz-von-Siebold-Stiftung, Band 4, 1992 (S. 63-88). Mūnchen: iudicium.

Trommsdorff, G. (1993b, in Vorb.). Zukunftsorientierung, Kontrollüberzeugung und Handeln. In E. Holzt \& J, Rinderspacher (Hrsg.), Erwartungen an die Zukunft. Frankfurt: Campus.

Weisz, J.R., Rothbaum, F.M., \& Blackbum, T.C. (1984). Standing out and standing in: The psychology of control in America and Japan. American Psychologist, 34, 955-969.

Zapf, W. (1993). Die DDR 1989/1990 - Zusammenbruch einer Sozialstruktur? In H. Joas \& M. Kohli (Hrsg.), Der Zusammenbruch der DDR (S.29-48). Frankfurt a.M.: Suhrkamp. 